\title{
Formaldehyde observations at XAO
}

\author{
Gang Wu, Jarken Esimbek, Jian-Jun Zhou and Wei-Guang Ji \\ Xinjiang Astronomical Observatory, CAS \\ Postbus 830011, 150 Science 1-Street, Urumuqi, China \\ email: wug@xao.ac.cn
}

\begin{abstract}
In recent years our team has performed $\mathrm{H}_{2} \mathrm{CO}\left(1_{10}-1_{11}\right)$ observations towards GMCs and HII regions with the Xinjiang Astronomical Observatory, CAS. Here, we provide a summary of these observations. More than 200 new formaldehyde sources are detected, 8 extended GMC have been mapped, kinetic distances, Galactic structure and a related discussion are provided.
\end{abstract}

Keywords. ISM: clouds - HII regions

\section{Introduction}

Owing to the extremely low excitation temperature (collision with neutral particles, proposed by Townes \& Cheung 1969) and relative low density, $\mathrm{H}_{2} \mathrm{CO}$ is commonly considered as a good tracer of low to warm temperatures and relatively dense nebulae.

\section{Observations}

We use the Nanshan $25 \mathrm{~m}$ antenna, operated by Xinjiang Astronomical Observatory, CAS, for our formaldehyde observations. The $\mathrm{H}_{110 \alpha}$ RRLs at $4874.1570 \mathrm{MHz}$ and the $\mathrm{H}_{2} \mathrm{CO}$ absorption lines at $4829.6594 \mathrm{MHz}$ are observed simultaneously. At this frequency the half power width of the main beam is about 10". A Digital Filter Bank spectrometer with 8192 channels is used, which results in a velocity resolution of $0.13 \mathrm{~km} \mathrm{~s}^{-1}$. The pointing and tracking accuracy is better than 20", and the beam efficiency is 0.65 . The system temperature is about 23K. DPFU (Degrees Per Flux Unit) is $0.116 \mathrm{~K} / \mathrm{Jy}$.

\section{Results and Summary}

1) more than 200 new formaldehyde sources are detected for the first time.

2) 8 extended star formation regions are mapped with $\mathrm{H}_{2} \mathrm{CO}$ absorption lines and $\mathrm{H}_{110 \alpha}$ RRLs. All the regions show that the formaldehyde distribution is similar to the large scale $\mathrm{CO}$ but has less correlation at small scales. Meanwhile, the distribution of $\mathrm{H}_{2} \mathrm{CO}$ also reveals a distribution similar to a $8.28 \mu \mathrm{m}$ MSX color map and CBT at $4.8 \mathrm{GHz}$.

3) We resolve the kinematic distance ambiguities for 14 HII regions and 20 intervening molecular clouds. These numbers suggest that UCHII regions are tightly confined to the Galactic plane. There is a good statistical relationship between the fluxes of $\mathrm{H}_{2} \mathrm{CO}$ and infrared $100 \mu \mathrm{m}$ for those HII regions with two lines detected. This suggests that there is a weak correlation between the continuum fluxes at $6 \mathrm{~cm}$ and infrared $100 \mu \mathrm{m}$.

\section{Reference}

Townes C. H. \& Cheung A. C., 1969, ApJ, 157, L103 